\title{
Respiratory Chain Complex Disorganization Impairs Mitochondrial and Cellular Integrity
}

\section{Phenotypic Variation in Cytochrome c Oxidase Deficiency}

\author{
Hideyuki Hatakeyama* ${ }^{* \dagger}$ and Yu-ichi Goto* ${ }^{* \dagger}$
}

From the Department of Mental Retardation and Birth Defect Research, ${ }^{*}$ National Institute of Neuroscience, and the Medical Genome Center, ${ }^{\ddagger}$ National Center of Neurology and Psychiatry, Tokyo; and AMED-CREST, Japan Agency for Medical Research and Development, Tokyo, Japan

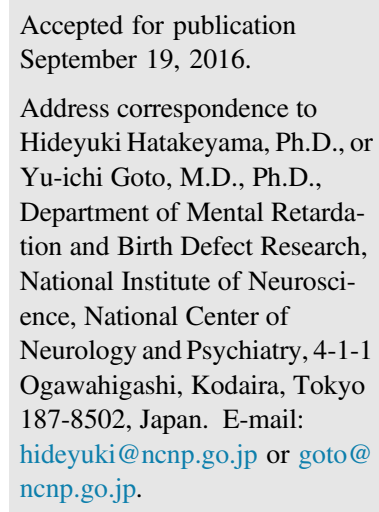

\begin{abstract}
The relationships between the molecular abnormalities in mitochondrial respiratory chain complexes and their negative contributions to mitochondrial and cellular functions have been proved to be essential for better understandings in mitochondrial medicine. Herein, we established the method to identify disease phenotypic differences among patients with muscle histopathological cytochrome $c$ oxidase (COX) deficiency, as one of the representative clinical features in mitochondrial diseases, by using patients' myoblasts that are derived from biopsied skeletal muscle tissues. We identified two obviously different severities in molecular diagnostic criteria of COX deficiency among patients: structurally stable, but functionally mild/moderate defect and severe functional defect with the disrupted COX holoenzyme structure. COX holoenzyme disorganization actually triggered several mitochondrial dysfunctions, including the decreased ATP level, the increased oxidative stress level, and the damaged membrane potential level, all of which lead to the deteriorated cellular growth, the accelerated cellular senescence, and the induced apoptotic cell death. Our cell-based in vitro diagnostic approaches would be widely applicable to understanding patient-specific pathomechanism in various types of mitochondrial diseases, including other respiratory chain complex deficiencies and other mitochondrial metabolic enzyme deficiencies. (Am J Pathol 2017, 187: 110-121; http://dx.doi.org/ 10.1016/j.ajpath.2016.09.003)
\end{abstract}

Cytochrome $c$ oxidase [COX; alias complex IV (CIV)] is a terminal protein in the mitochondrial electron transport system with oxidative phosphorylation and comprises its 13 structural subunits. The three largest, most hydrophobic catalytic core subunits are encoded in mitochondrial DNA (mtDNA), and the others are encoded in nuclear DNA (nDNA). In addition, COX also requires several nDNAencoded assembly factors for its holoenzyme organization and maintenance. COX deficiency is widely recognized as one of the representative clinical phenotypes in mitochondrial diseases and presents muscle histopathological diversity among patients (focally, diffusely, or completely deficient). Although disease-causative mutations in nDNAencoded assembly factors are mostly inherited as autosomal recessive, ${ }^{1}$ only a few detrimental mutations in nDNA-encoded COX structural subunits have been reported. ${ }^{2,3}$ Other genetic defects in mtDNA-encoded COX structural subunits ${ }^{4-10}$ or in several mitochondrial tRNA genes are also responsible for COX deficiency; moreover, infantile reversible COX deficiency (alias reversible infantile respiratory chain deficiency), which is caused by homoplasmic m.14674T $>\mathrm{C}$ or $\mathrm{T}>\mathrm{G}$ mutations in $M T-T E$ gene, has recently been identified as a new disease subtype with rare, distinct disease outcome. ${ }^{11,12}$ To date, the relationships between pathogenic mutations in COX-

\footnotetext{
Supported in part by Japan Society for the Promotion of Science grant-inaid for Young Researcher B 20790760 (H.H.); a Ministry of Health, Labour, and Welfare, Japan grant-in-aid of the Research on Intractable Diseases (Mitochondrial Disorder) (Y.G.); and Japan Agency for Medical Research and Development AMED-CREST.
}

Disclosures: None declared. 
associating components and the aberrant COX holoenzyme organization become evident at a molecular level. However, there still remains no reasonable explanation how such gene-specific defects actually affect widespread mitochondrial and cellular functions, resulting in the variation and the severity of disease phenotypes at tissue and organ levels.

To overcome this problem, the use of cells derived from the affected tissues and organs is advantageous, because such cells faithfully recapitulate cell type-specific pathophysiology in a patient-specific manner. Herein, we established the method to identify disease phenotypic differences in patients exhibiting mitochondrial diseases by using a comprehensive functional analysis at mitochondrion and cell levels. We demonstrated that severely disrupted COX holoenzyme integrity (its function and structure) actually triggered several mitochondrial dysfunctions, including the decreased ATP level, the increased oxidative stress level, and the damaged membrane potential level, followed by the injured cellular homeostasis like the deteriorated cellular growth, the accelerated cellular senescence, and the induced apoptotic cell death. Therefore, COX holoenzyme disorganization determines the variation and the severity in clinical phenotypes of patients exhibiting mitochondrial diseases with muscle histopathological COX deficiency, and our proposed molecular diagnostic criteria may also be suggestive for effectively exploring disease-causative genetic defects, which are responsible for patient-specific pathology.

\section{Materials and Methods}

\section{Patients}

This study was approved by the institutional review board of the National Center of Neurology and Psychiatry and was stringently conducted in accordance with the ethical principles of the Declaration of Helsinki. Patient skeletal muscle biopsy was performed for diagnostic purposes only after we received written informed consent. Note that 10 control subjects were also used in this study.

\section{mtDNA Mutation Analysis}

A long PCR-based whole mtDNA sequence in each patient was performed to eliminate any adverse results associating with pseudosequences in nDNA, as described elsewhere ${ }^{13}$ with modifications: Extracted DNA from cultured patients' myoblasts (100 ng) was amplified by mtDNA-specific longrange PCR and the following mtDNA-specific nested PCR using a thermal cycler (GeneAmp PCR System 9700; Applied Biosystems, Waltham, MA). The amplified mtDNA fragments were sequenced using DNA analyzer (ABI PRISM 3130xl; Applied Biosystems). The obtained mtDNA sequence data in each patient were compared with the WEB databases of Human Mitochondrial Genome Database (MITOMAP) and Human Mitochondrial Genome Polymorphism (mtSNP) ${ }^{14}$ to find any genetic variants.

\section{RT-PCR}

One-step RT-PCR was performed with the PrimeScript II High Fidelity RT-PCR kit (TaKaRa Bio, Shiga, Japan), according to the manufacturer's instructions. Extracted total RNA from cultured patients' myoblasts (100 ng) was applied for RT-PCR using a thermal cycler (GeneAmp PCR system 9700; Applied Biosystems). Amplified PCR products were electrophoresed, stained with ethidium bromide, and detected using UV transilluminator (GelDocIt Imaging System; UVP, Upland, CA).

Primers used were as follows: MT-CO1, 5'-TTAGCTGACTCGCCACACTCC-3' (forward) and 5'-AGTCAGGCCACCTACGGTGA-3' (reverse); MT-CO2, 5' -CTCATGAGCTGTCCCCACATTAG-3' (forward) and 5'-TTGACCGTAGTATACCCCCGG-3' (reverse); COX4, 5'-CGGCAGAATGTTGGCTACCA- $3^{\prime}$ (forward) and $5^{\prime}$-AGCGAAAAGTCTTCGCTCTTCAC-3' (reverse); COX5B, $5^{\prime}$-TGGCATCTGGAGGTGGTGTT- $3^{\prime}$ (forward) and $5^{\prime}$-TGCCTGAAGCTCCCTTTGG-3' (reverse); and GAPDH, 5'-CAATGACCCCTTCATTGACCTC-3' (forward) and 5'-CTCGCTCCTGGAAGATGGTG-3' (reverse).

\section{Cell Culture}

Small portions of biopsied skeletal muscle tissues from the patients' biceps brachii were minced with surgical scissors and forceps, enzymatically digested with collagenase-trypsin solution [400 $\mu \mathrm{g} / \mathrm{mL}$ collagenase (Wako, Osaka, Japan), $5 \times$ trypsin-EDTA (Gibco, Waltham, MA)] at $37^{\circ} \mathrm{C}$ for 1 hour, and centrifuged at $200 \times g$ for 5 minutes to collect myoblasts. Cells were resuspended and seeded onto tissue culture dishes and were maintained at $37^{\circ} \mathrm{C}$ under humidified atmosphere of $5 \% \mathrm{CO}_{2}$. Myoblast culture medium used was as follows: Dulbecco's modified Eagle's medium with F12 nutrient mixture (Gibco) supplemented with $20 \%$ fetal bovine serum (Gibco), $100 \mathrm{U} / \mathrm{mL}$ penicillin (Gibco), and $100 \mu \mathrm{g} / \mathrm{mL}$ streptomycin (Gibco). During primary culture, $0.5 \mu \mathrm{g} / \mathrm{mL}$ MC210 (DS Pharm, Osaka, Japan) as a mycoplasmacidal reagent and $2.5 \mu \mathrm{g} / \mathrm{mL}$ fungizone (Gibco) as a fungicidal reagent were also added into myoblast culture medium.

For cellular proliferation experiment, patients' myoblasts $\left(100 \mathrm{cells} / \mathrm{mm}^{2}\right)$ were seeded onto $96-$ well culture plates and were maintained at $37^{\circ} \mathrm{C}$ under humidified atmosphere of $5 \% \mathrm{CO}_{2}$. After 3 days in culture, cells were treated with bromodeoxyuridine chemiluminescence-based cell proliferation enzyme-linked immunosorbent assay kit (Roche, Basel, Switzerland), according to the manufacturer's instructions, and cellular proliferation potential was measured on chemiluminescent multiwell plate reader (Centro LB 960; Berthold Technologies, Bad Wildbad, Germany).

For cellular growth experiment, patients' myoblasts $\left(50\right.$ cells $\left./ \mathrm{mm}^{2}\right)$ were seeded onto 6-well culture plates and were maintained at $37^{\circ} \mathrm{C}$ under humidified atmosphere of $5 \% \mathrm{CO}_{2}$. Cells were observed under phase contrast microscope (IX71 System; Olympus, Tokyo, Japan) at 
predetermined time intervals. Cell number per unit area was randomly counted and averaged in each sample. Cellular doubling time was also estimated at logarithmic proliferation stage.

For cellular senescence detection, patients' myoblasts (100 cells $/ \mathrm{mm}^{2}$ ) were seeded onto 4-well culture slides and were maintained at $37^{\circ} \mathrm{C}$ under humidified atmosphere of $5 \% \mathrm{CO}_{2}$. After 3 days in culture, cells were treated with senescenceassociated $\beta$-galactosidase staining kit (Cell Signaling Technology, Danvers, MA), according to the manufacturer's instructions, and senescent cells were observed under optical microscope (BX50 System; Olympus).

For apoptotic cell death detection, patients' myoblasts $\left(100\right.$ cells $\left./ \mathrm{mm}^{2}\right)$ were seeded onto 6-well culture plates and were maintained at $37^{\circ} \mathrm{C}$ under humidified atmosphere of $5 \% \mathrm{CO}_{2}$. After 3 days in culture, cells were treated with caspase-3 detection kit (Biotium, Fremont, CA), according to the manufacturer's instructions, and apoptotic cell death was observed under fluorescent microscope (IX71 System; Olympus).

For terminal differentiation into myotubes, patients' myoblasts $\left(200\right.$ cells $\left./ \mathrm{mm}^{2}\right)$ were seeded onto 6-well culture plates and were maintained at $37^{\circ} \mathrm{C}$ under humidified atmosphere of $5 \% \mathrm{CO}_{2}$. After 3 days in culture, culture medium was switched to differentiation medium (Cell Applications, San Diego, CA) supplemented with $100 \mathrm{U} / \mathrm{mL}$ penicillin (Gibco) and $100 \mu \mathrm{g} / \mathrm{mL}$ streptomycin (Gibco), and cells were maintained for 2 weeks.

\section{Cytochemistry}

Patients' myoblasts (100 cells $/ \mathrm{mm}^{2}$ ) were seeded onto 4-well culture slides and were maintained at $37^{\circ} \mathrm{C}$ under humidified atmosphere of $5 \% \mathrm{CO}_{2}$. After 3 days in culture, cells were stained with COX reaction buffer (pH 5.5; $100 \mathrm{mmol} / \mathrm{L}$ sodium acetate, $0.1 \% \mathrm{MnCl}_{2}, 0.001 \% \mathrm{H}_{2} \mathrm{O}_{2}$, and $10 \mathrm{mmol} / \mathrm{L}$ diaminobenzidine) at $37^{\circ} \mathrm{C}$ for 1 hour and were incubated with $1 \% \mathrm{CuSO}_{4}$ at $37^{\circ} \mathrm{C}$ for 5 minutes. Cell nuclei were costained with hematoxylin. Stained cells were rinsed, fixed, and dehydrated according to standard histological protocol. Samples were sealed with cover glass and were observed under optical microscope (BX50 System; Olympus).

\section{Immunocytochemistry}

Cultured patients' myoblasts and differentiated myotubes were fixed, permeabilized, and blocked according to standard immunocytochemical protocol. Primary antibody probing was performed at room temperature for 90 minutes. Secondary antibody probing was performed with $2.5 \mu \mathrm{g} / \mathrm{mL}$ Alexa Fluor 568 (Molecular Probes, Waltham, MA) at room temperature for 45 minutes. Stained cells were observed under fluorescent microscope (IX71 System; Olympus).

Primary antibodies used were as follows: $2.5 \mu \mathrm{g} / \mathrm{mL}$ anti-MT-CO1 (Molecular Probes), $2.5 \mu \mathrm{g} / \mathrm{mL}$ anti-COX4 (Molecular Probes), $0.5 \mu \mathrm{g} / \mathrm{mL}$ anti-SDHA (Molecular
Probes), $5 \mu \mathrm{g} / \mathrm{mL}$ anti-myogenin (Abcam, Cambridge, UK), and $5 \mu \mathrm{g} / \mathrm{mL}$ anti-actin, $\alpha 1$, skeletal muscle (Abcam).

\section{Mitochondrial Enzymatic Activity}

Enzymatic activities for individual mitochondrial respiratory chain complexes were analyzed as described elsewhere ${ }^{15}$ with modifications: Cultured and harvested patients' myoblasts (100,000 cells/assay) were permeabilized with $0.1 \%$ digitonin at room temperature for 1 minute with gentle pipetting and were used for the experiment. A spectrophotometer equipped with a thermostated unit (U-2010; Hitachi, Tokyo, Japan) was used, and a baseline calibration was done before each measurement.

For complex I activity measurement, permeabilized cells were added into reaction buffer $(\mathrm{pH} 7.4 ; 50 \mathrm{mmol} / \mathrm{L}$ Tris-HCl, $250 \mathrm{mmol} / \mathrm{L}$ sucrose, $1 \mathrm{mmol} / \mathrm{L}$ EDTA, $10 \mu \mathrm{mol} / \mathrm{L}$ decylubiquinone, $50 \mu \mathrm{mol} / \mathrm{L}$ NADH, $5 \mu \mathrm{g} / \mathrm{mL}$ antimycin $\mathrm{A}$, and $2 \mathrm{mmol} / \mathrm{L} \mathrm{KCN}$ ) and were incubated in quartz cuvette at $37^{\circ} \mathrm{C}$. Complex I activity was monitored by time-dependent absorbance alterations.

For complex II activity measurement, permeabilized cells were added into reaction buffer $(\mathrm{pH} 7.4 ; 50 \mathrm{mmol} / \mathrm{L}$ potassium phosphate, $20 \mathrm{mmol} / \mathrm{L}$ succinate, $50 \mu \mathrm{mol} / \mathrm{L}$ 2,6-dichlorophenolindophenol, $50 \mu \mathrm{mol} / \mathrm{L}$ decylubiquinone, $5 \mu \mathrm{g} / \mathrm{mL}$ rotenone, $5 \mu \mathrm{g} / \mathrm{mL}$ antimycin $\mathrm{A}$, and $2 \mathrm{mmol} / \mathrm{L}$ $\mathrm{KCN}$ ) and were incubated in quartz cuvette at $37^{\circ} \mathrm{C}$. Complex II activity was monitored by time-dependent absorbance alterations.

For complex III activity measurement, permeabilized cells were added into reaction buffer $[\mathrm{pH} 7.4 ; 50 \mathrm{mmol} / \mathrm{L}$ Tris-HCl, $250 \mathrm{mmol} / \mathrm{L}$ sucrose, $1 \mathrm{mmol} / \mathrm{L}$ EDTA, $50 \mu \mathrm{mol} / \mathrm{L}$ cytochrome $c, 50 \mu \mathrm{mol} / \mathrm{L}$ decylubiquinol (reduced form of decylubiquinone), and $2 \mathrm{mmol} / \mathrm{L} \mathrm{KCN}$ ] and were incubated in quartz cuvette at $37^{\circ} \mathrm{C}$. Complex III activity was monitored by time-dependent absorbance alterations.

For complex IV activity measurement, permeabilized cells were added into reaction buffer $[\mathrm{pH} 7.4 ; 10 \mathrm{mmol} / \mathrm{L}$ potassium phosphate and $25 \mu \mathrm{mol} / \mathrm{L}$ ferrocytochrome $c$ (reduced form of cytochrome $c$ )] and were incubated in quartz cuvette at $37^{\circ} \mathrm{C}$. Complex IV activity was monitored by time-dependent absorbance alterations.

For citrate synthase activity measurement, permeabilized cells were added into reaction buffer $[\mathrm{pH} 8.0 ; 125 \mathrm{mmol} / \mathrm{L}$ Tris-HCl, $300 \mu \mathrm{mol} / \mathrm{L}$ acetyl-CoA, $100 \mu \mathrm{mol} / \mathrm{L} \quad 5,5^{\prime}-$ dithiobis (2-nitrobenzoic acid), and $500 \mu \mathrm{mol} / \mathrm{L}$ oxaloacetate] and were incubated in quartz cuvette at $37^{\circ} \mathrm{C}$. Citrate synthase activity was monitored by time-dependent absorbance alterations.

\section{Electrophoretic Protein Separation}

SDS-PAGE, blue native PAGE (BN-PAGE), and twodimensional BN-PAGE/SDS-PAGE were performed as described elsewhere ${ }^{16,17}$ with modifications, respectively: Cultured and harvested patients' myoblasts were resuspended 
in isolation buffer $(\mathrm{pH} 7.4 ; 210 \mathrm{mmol} / \mathrm{L}$ mannitol, $70 \mathrm{mmol} / \mathrm{L}$ sucrose, $1 \mathrm{mmol} / \mathrm{L}$ EGTA, and $5 \mathrm{mmol} / \mathrm{L}$ HEPES) and were homogenated on ice. Cell lysates were centrifuged to isolate mitochondrial proteins. Obtained mitochondrial proteins were quantified by Bradford assay, and a calibration curve was generated using several known concentrations of bovine serum albumin.

For SDS-PAGE, isolated mitochondrial proteins $(100 \mu \mathrm{g})$ were solubilized with $0.5 \%$ SDS containing $50 \mathrm{mmol} / \mathrm{L}$ dithiothreitol at $70^{\circ} \mathrm{C}$ for 10 minutes and were used for the experiment. Electrophoresis was performed on $4 \%$ to $12 \%$ NuPAGE polyacrylamide gel (Invitrogen, Waltham, MA) at room temperature under $200-\mathrm{V}$ constant.

For BN-PAGE, isolated mitochondrial proteins $(100 \mu \mathrm{g})$ were solubilized with either $0.5 \% n$-dodecyl- $\beta$-D-maltoside (individual complexes detection) or $1 \%$ digitonin (supercomplexes detection) on ice for 30 minutes and were used for the experiment. Electrophoresis was performed on 3\% to $12 \%$ NativePAGE polyacrylamide gel (Invitrogen) at $4^{\circ} \mathrm{C}$ under $150-\mathrm{V}$ constant for 30 minutes, then resumed at $4^{\circ} \mathrm{C}$ under $250-\mathrm{V}$ constant.

\section{Western Blot for Immunodetection}

Electrophoresed gels were blotted onto polyvinylidene difluoride membranes using iBlot transfer system (Invitrogen), according to the manufacturer's instructions. Blotted polyvinylidene difluoride membranes were blocked at room temperature for 30 minutes. Primary antibody probing was performed at room temperature for 90 minutes. Secondary antibody probing was performed with chromogenic antibody detection kit (WesternBreeze; Invitrogen), according to the manufacturer's instructions.

For SDS-PAGE and immunodetection, primary antibodies used were as follows: $0.5 \mu \mathrm{g} / \mathrm{mL}$ anti-SDHA (Molecular Probes), $2.5 \mu \mathrm{g} / \mathrm{mL}$ anti-MT-CO1 (Molecular Probes), $2.5 \mu \mathrm{g} / \mathrm{mL}$ anti-MT-CO2 (Molecular Probes), 2.5 $\mu \mathrm{g} / \mathrm{mL}$ anti-COX4 (Molecular Probes), $2.5 \mu \mathrm{g} / \mathrm{mL}$ antiCOX5B (Molecular Probes), and $2.5 \mu \mathrm{g} / \mathrm{mL}$ anti-COX6B (Molecular Probes).

For BN-PAGE and immunodetection, primary antibodies used were as follows: $0.5 \mu \mathrm{g} / \mathrm{mL}$ anti-NDUFA9 (Molecular Probes), $0.5 \mu \mathrm{g} / \mathrm{mL}$ anti-SDHA (Molecular Probes), $0.5 \mu \mathrm{g} / \mathrm{mL}$ anti-UQCRC2 (Molecular Probes), 2.5 $\mu \mathrm{g} / \mathrm{mL}$ anti-MT-CO1 (Molecular Probes), and $0.5 \mu \mathrm{g} / \mathrm{mL}$ anti-ATP5B (Molecular Probes).

\section{Detection of Intracellular ATP}

Cultured and harvested patients' myoblasts (100 cells/assay) were applied for the measurements. Cells were treated with rLuciferase/Luciferin chemiluminescence-based ATP detection kit (Promega, Fitchburg, WI), according to the manufacturer's instructions, and intracellular ATP amount was measured on chemiluminescent multiwell plate reader (Centro LB 960; Berthold Technologies). A calibration curve was generated using several known concentrations of ATP.

\section{Detection of Mitochondrial 0xidative Stress and Mitochondrial Membrane Potential}

Quantitative fluorometry was performed as follows: Patients' myoblasts $\left(100\right.$ cells $\left./ \mathrm{mm}^{2}\right)$ were seeded onto 96 -well culture plates and were maintained at $37^{\circ} \mathrm{C}$ under humidified atmosphere of $5 \% \mathrm{CO}_{2}$. After 3 days in culture, cells were stained at $37^{\circ} \mathrm{C}$ for 1 hour. Stained cells were rinsed and measured on fluorescent multiwell plate reader (ARVO SX; Perkin Elmer, Waltham, MA), first at excitation/emission of 545/595 nm (red fluorescence) and then sequentially at excitation/ emission of 485/535 nm (green fluorescence).

Fluorescent imaging was performed as follows: Patients' myoblasts $\left(100\right.$ cells $\left./ \mathrm{mm}^{2}\right)$ were seeded onto 6-well culture plates and were maintained at $37^{\circ} \mathrm{C}$ under humidified atmosphere of $5 \% \mathrm{CO}_{2}$. After 3 days in culture, cells were stained at $37^{\circ} \mathrm{C}$ for 1 hour. Stained cells were rinsed and observed under fluorescent microscope (IX71 System; Olympus).

Fluorescent dyes used were as follows: $0.25 \mu \mathrm{g} / \mathrm{mL}$ MitoTracker Green (Molecular Probes), $0.25 \mu \mathrm{g} / \mathrm{mL}$ MitoSOX Red (Molecular Probes), and $0.25 \mu \mathrm{g} / \mathrm{mL}$ JC-1 (Molecular Probes).

\section{Results}

\section{Molecular Pathogenic Variation among Patients with COX Deficiency}

We examined seven mitochondrial disease patients with muscle histopathological COX deficiency, all of whom carry no detrimental mutation on entire mtDNA sequence (Supplemental Tables S1-S7). In addition, no typical pathological abnormality was observed in all patient-derived skeletal muscle tissues other than COX deficiency (Supplemental Figure S1). On muscle histopathological COX staining, the numerical and distributional variation of COX-negative muscle fibers was observed among patients' tissues (Figure 1A); Patients 5 and 6 also exhibited completely deficient COX activity. On cytochemical COX staining, a wide variety of the decreased COX activity was also observed among patients' myoblasts (Figure 1A), showing a trend similar to muscle histopathological COX staining. BN-PAGE and immunodetection indicated that the apparently diminished band corresponding to COX holoenzyme was detected only in Patients 5 and 6, whereas the other patients showed stable COX holoenzyme organization (Figure 1B); Patients 5 and 6 also showed no COXcontaining respiratory chain supramolecular architectures that were essential for efficient ATP production in the mitochondrial electron transport system (Figure 1C). The assembly of the other respiratory chain complexes was unaffected in all patients. We therefore defined Patients 5 

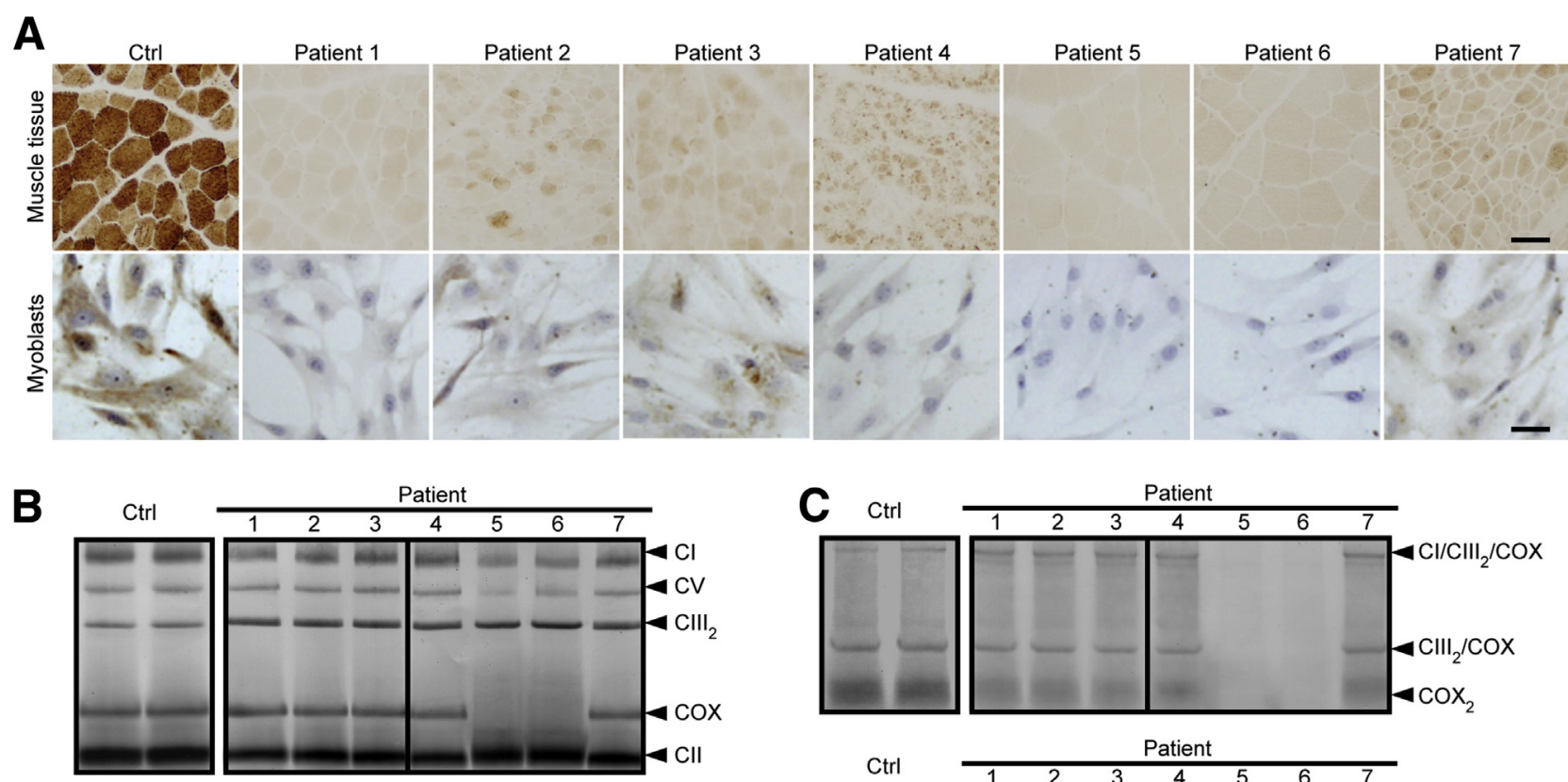

COX defect (+): Patients \#5, \#6

coX defect (-): Patients \#1, \#2, \#3, \#4, \#7
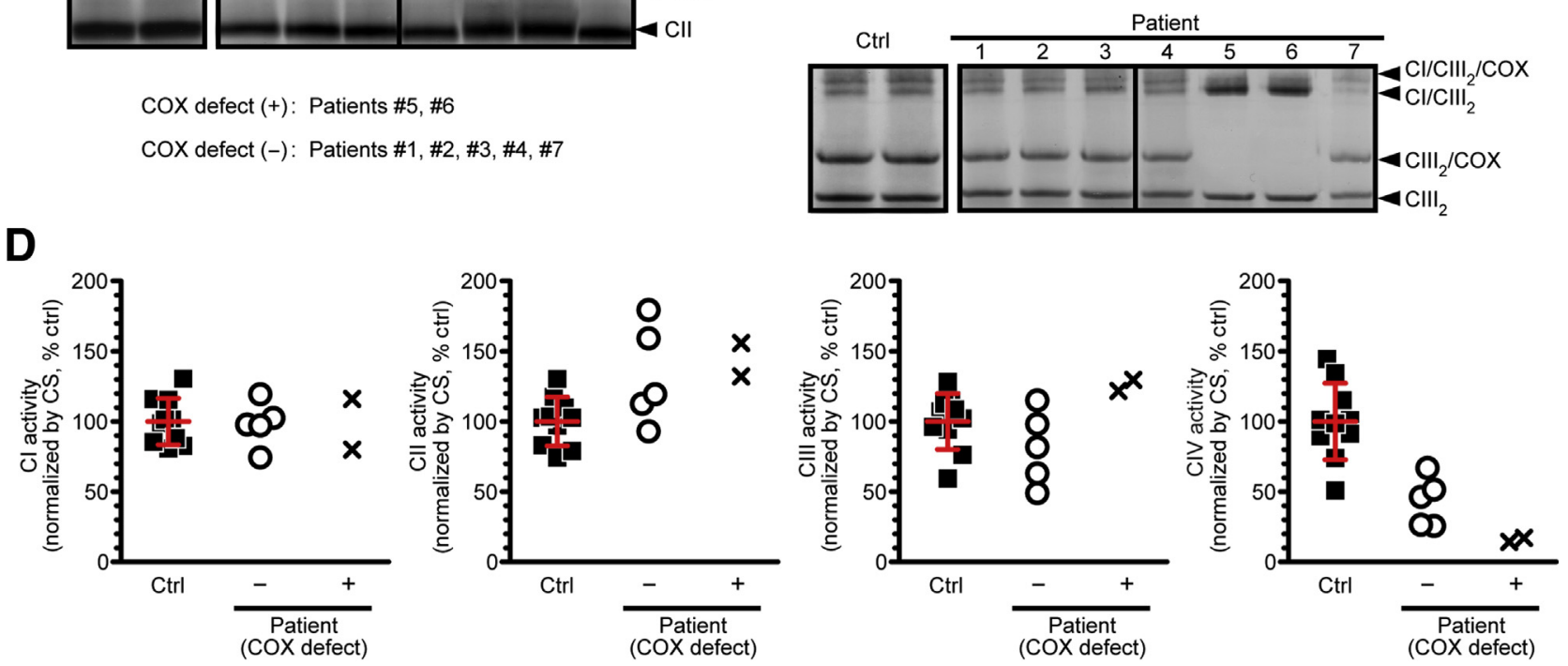

Figure 1 Molecular pathogenic variation among patients with COX deficiency. A: Representative images of COX staining for frozen section of skeletal muscle specimens (top row) and for cultured myoblasts (bottom row) of both controls (Ctrl) and patients. On cytochemical coX staining for cultured myoblasts, all samples were stained simultaneously with the same period. Cell nuclei were costained with hematoxylin. B: Immunodetection of individual respiratory chain complexes (CI, CII, CIII, COX, and CV) by BN-PAGE for the same amount of isolated mitochondrial proteins from both controls and patients. Primary antibodies used were as follows: anti-NDUFA9 (CI), anti-SDHA (CII), anti-UQCRC2 (CIII), anti-MT-C01 (COX), and anti-ATP5B (CV). All samples were assayed at least in duplicate. C: Immunodetection of respiratory chain supercomplexes by BN-PAGE for the same amount of isolated mitochondrial proteins from both controls and patients. Primary antibodies used were as follows: anti-MT-C01 (COX) (top row) and anti-UQCRC2 (CIII) (bottom row). All samples were assayed at least in duplicate. D: Enzymatic activities of individual mitochondrial respiratory chain complexes for cultured and harvested myoblasts of both controls and patients. Citrate synthase (CS) activity, as an internal marker in mitochondrial functions, was used for normalization in each sample. All samples were measured at least in duplicate and averaged. The error bars indicate means \pm SD of controls (D). $n=10$ (D, controls); $n=5[\mathbf{D}$, COX defect $(-)] ; n=2[\mathbf{D}$, COX defect $(+)]$. Scale bars $=50 \mu \mathrm{m}(\mathbf{A})$.

and 6 lacking COX holoenzyme as COX defect $(+)$ and the other patients exhibiting stable COX holoenzyme as COX defect (-). Enzymatic activities of mitochondrial respiratory chain complexes revealed that COX activity in all patients was significantly decreased when compared with controls (Figure 1D); Patients 5 and 6 as COX defect (+) also displayed lower biochemical COX function. The other respiratory chain complex activities in all patients were almost within normal range, except for relatively higher complex II activity, probably because of functional compensation in mitochondrial oxidative phosphorylation. From molecular diagnostic aspects in mitochondrial respiratory chain complexes, we concluded that all patients used in this study must be isolated COX deficiency.

Two-dimensional BN-PAGE/SDS-PAGE implied that the apparent loss of COX holoenzyme found only in Patients 5 and 6 was because of drastically decreased amounts of all COX structural subunits when compared with other 

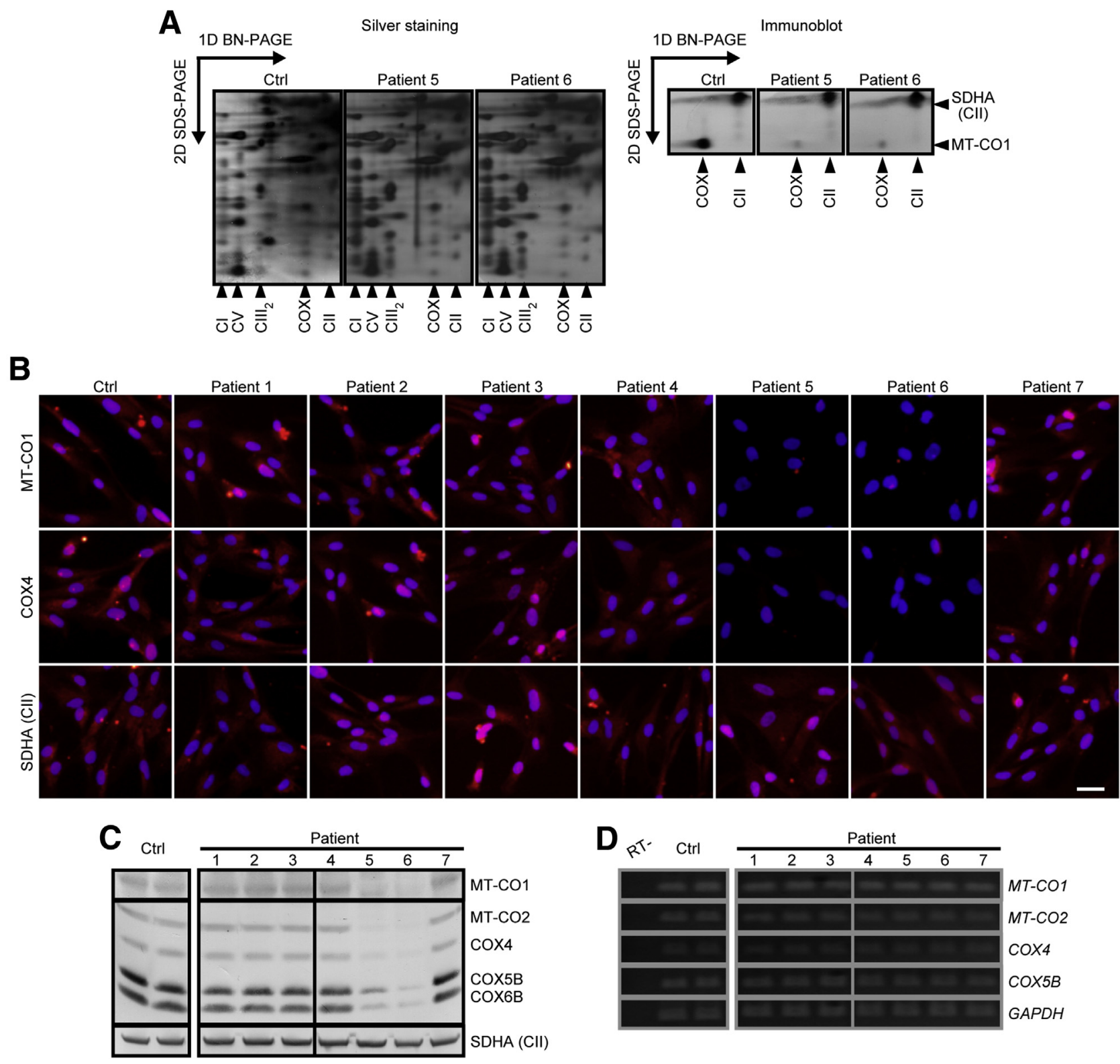

D
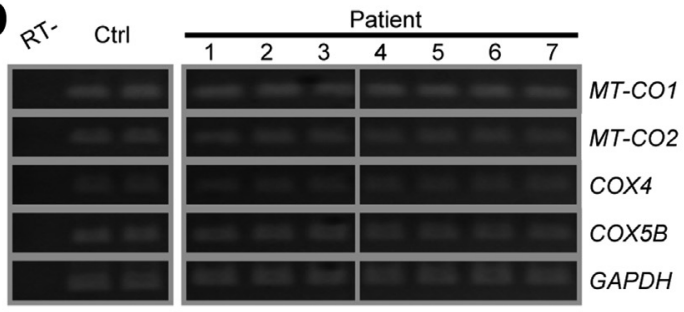

Figure 2 COX holoenzyme disorganization, found only in Patients 5 and 6, originates in the decreased protein levels, but not in mRNA levels, of each structural subunit. A: Visualization of all structural components in respiratory chain complexes by two-dimensional blue native PAGE (BN-PAGE)/SDS-PAGE and silver staining for the same amount of isolated mitochondrial proteins from both controls (Ctrl) and Patients 5 and 6 as COX defect (+). Immunodetection against MT-CO1 (COX) and SDHA (CII) was also shown. All samples were assayed at least in duplicate. B: Representative images of immunocytochemistry against MT-C01 [COX, mitochondrial DNA (mtDNA) encoded], COX4 [COX, nuclear DNA (nDNA) encoded], and SDHA (CII) for cultured myoblasts of both controls and patients. Cell nuclei were costained with Hoechst 33342 (blue). C: Protein expression levels in COX structural subunits of MT-C01 (mtDNA encoded), MTC02 (mtDNA encoded), COX4 (nDNA encoded), COX5B (nDNA encoded), and COX6B (nDNA encoded) by SDS-PAGE and immunodetection for the same amount of isolated mitochondrial proteins from both controls and patients. SDHA (CII) was used as an internal marker. All samples were assayed at least in duplicate. D: mRNA expression levels in COX structural subunits of MT-CO1 (mtDNA encoded), MT-CO2 (mtDNA encoded), COX4 (nDNA encoded), COX5B (nDNA encoded) by RT-PCR for the same amount of extracted total RNA from both controls and patients. GAPDH was used as an internal marker. RT- indicates without the addition of reverse transcriptase in RT-PCR. All samples were assayed at least in duplicate. Scale bar $=50 \mu \mathrm{m}$ (B).

respiratory chain complex components (Figure 2A); it was consistent with the results of immunocytochemistry against both COX structural subunits of mtDNA-encoded MT-CO1 and nDNA-encoded COX4 (Figure 2B). In fact, significantly lower protein expression levels of several COX structural subunits were confirmed only in Patients 5 and 6 when compared with controls and the other patients exhibiting stable COX holoenzyme organization (Figure 2C). However, no significant alteration in mRNA expression levels was observed in all patients when compared with controls (Figure 2D). These results suggest that COX holoenzyme disorganization, found only in Patients 5 and 6 , originates in the decreased protein levels, but not in mRNA levels, of each COX structural subunit. 
Severely Impaired COX Holoenzyme Integrity Triggers the Deteriorated Mitochondrial and Cellular Homeostasis, but Does Not Affect Skeletal Muscle Development

To further investigate the influences of severely impaired COX holoenzyme integrity on mitochondrial and cellular homeostasis, we added cell-based functional analysis in all patients. The decreased ATP level was observed only in Patients 5 and 6 as COX defect $(+)$ when compared with controls and the other patients exhibiting stable COX holoenzyme organization (Figure 3A). Interestingly, the increased oxidative stress level (Figure 3, B and D) and the damaged membrane potential level (Figure 3, C and E) were both markedly detected only in Patients 5 and 6 as COX defect $(+)$, whereas the other patients showed no significant alteration in mitochondrial functions when compared with those of controls. These results demonstrate that COX holoenzyme disorganization can strongly induce several mitochondrial dysfunctions.

Among patients' myoblast lines, Patients 5 and 6 as COX defect $(+)$ exhibited significantly deteriorated proliferative potential in living cells (Figure 4A); it was consistent with the results of growth rate (Figure 4B) and doubling time (Figure 4B). Remarkably, some senescence-associated $\beta$-galactosidase-positive senescent cells (Figure 4C) and caspase 3-positive apoptotic cells (Figure 4D) were also detected only in Patients 5 and 6 as COX defect $(+)$, even
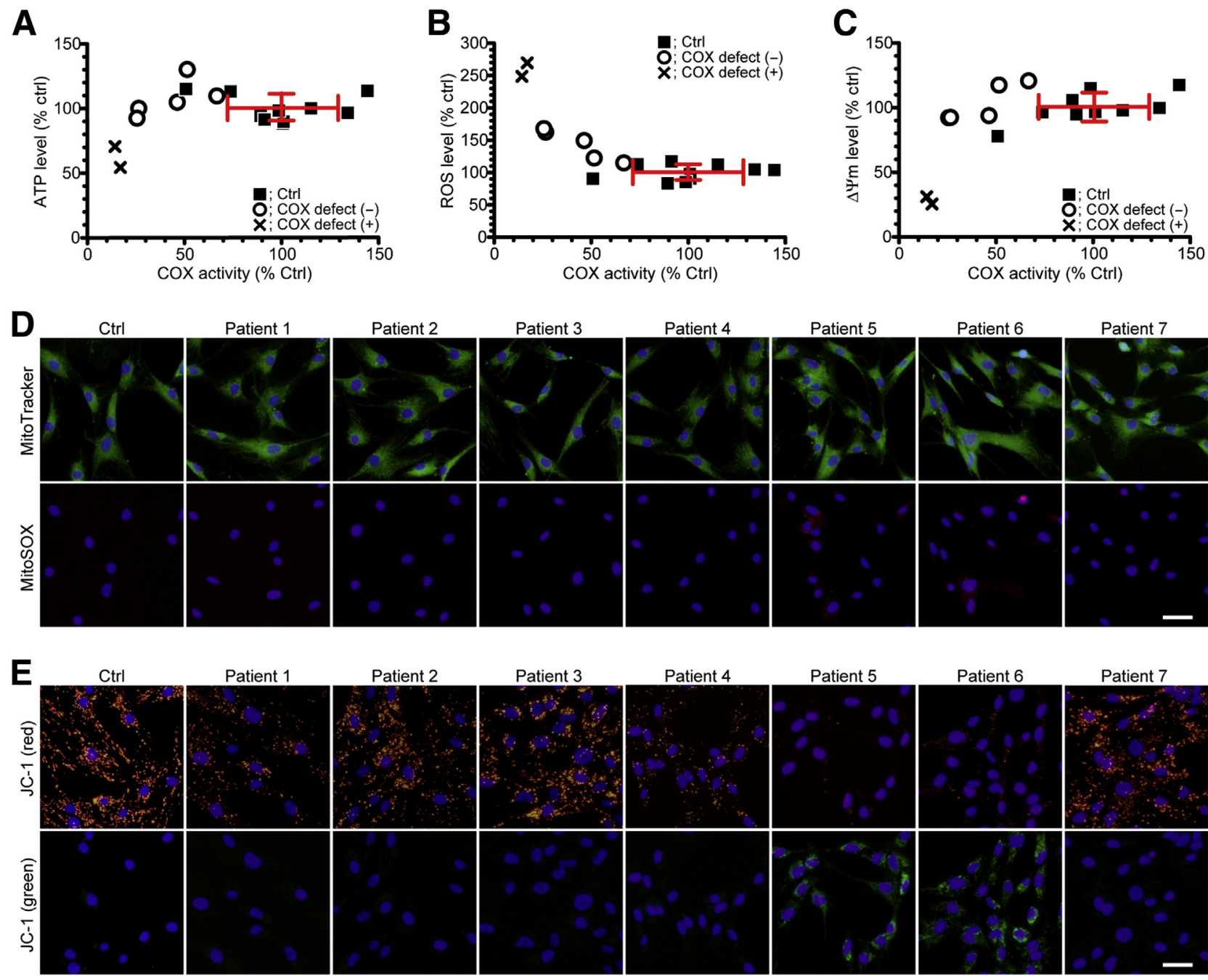

Patient 7

Figure 3 Severely impaired COX holoenzyme integrity triggers mitochondrial dysfunctions. A: Relationship between COX activity and ATP level for cultured and harvested myoblasts of both controls (Ctrl) and patients. All samples were measured at least in duplicate and averaged. B: Relationship between COX activity and oxidative stress [reactive oxygen species (ROS)] level for cultured myoblasts of both controls and patients. All samples were measured at least in duplicate and averaged. C: Relationship between COX activity and membrane potential ( $\Delta \Psi \mathrm{m})$ level for cultured myoblasts of both controls and patients. All samples were measured at least in duplicate and averaged. D: Representative images of the intracellular localization of MitoTracker (green) or MitoSOX (red) for cultured myoblasts of both controls and patients. Cell nuclei were costained with Hoechst 33342 (blue). E: Representative images of the intracellular localization of JC-1 monomer (green) or aggregates (red) for cultured myoblasts of both controls and patients. Cell nuclei were costained with Hoechst 33342 (blue). The error bars indicate means \pm SD of controls $(\mathbf{A}-\mathbf{C}) . n=10(\mathbf{A}-\mathbf{C}$, controls $) ; n=5[\mathbf{A}-\mathbf{C}, \mathbf{C O X}$ defect $(-)] ; n=2[\mathbf{A}-\mathbf{C}, \mathbf{C O X}$ defect $(+)]$. Scale bars $=50 \mu \mathrm{m}$ (D and $\mathbf{E})$. 

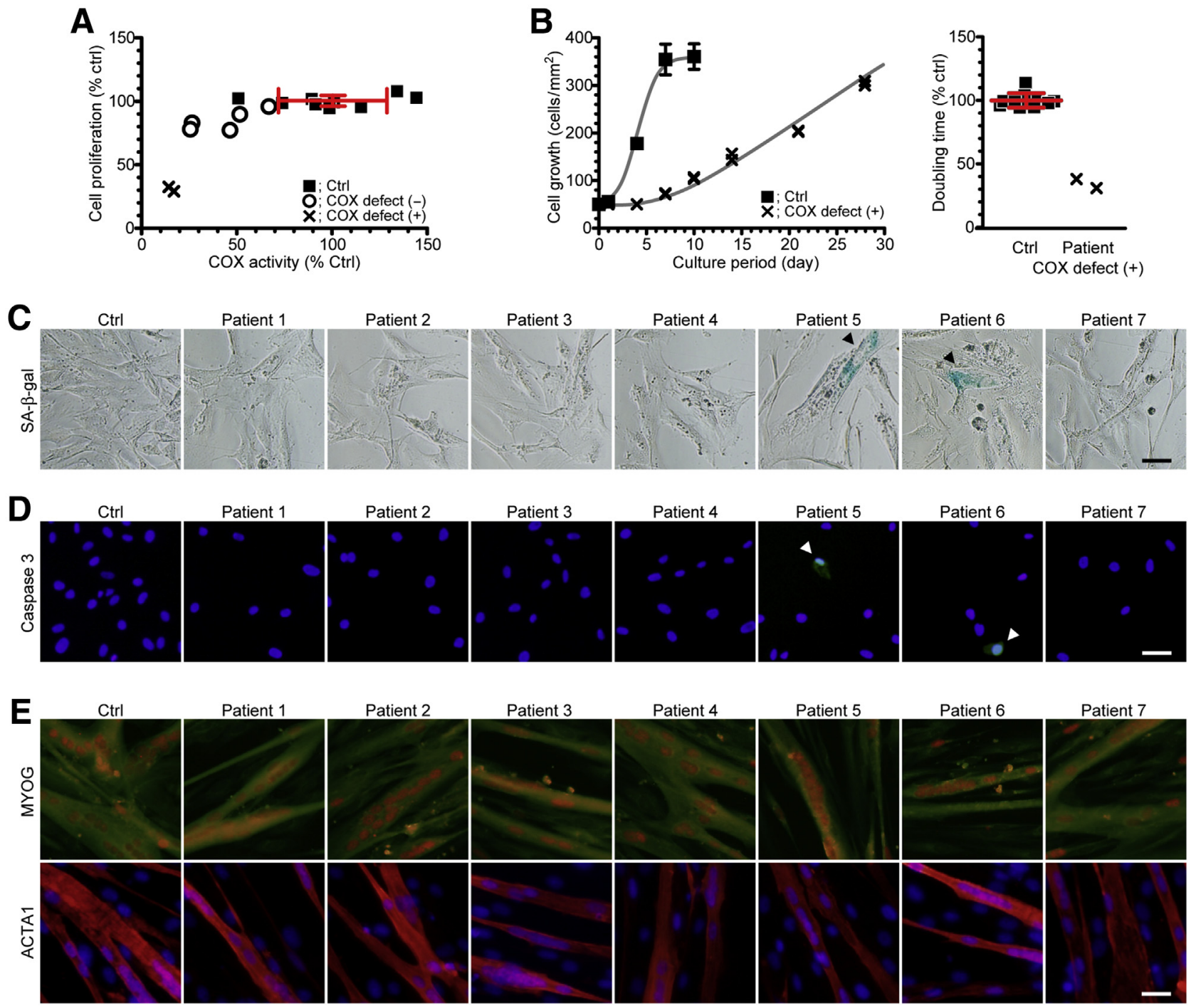

Figure 4 Severely impaired COX holoenzyme integrity also induces cellular dysfunctions, but does not affect skeletal muscle development. A: Relationship between COX activity and cellular proliferation potential (bromodeoxyuridine assay) for cultured myoblasts of both controls (Ctrl) and patients. All samples were measured at least in duplicate and averaged. B: Cellular growth rate for cultured myoblasts of both controls and Patients 5 and 6 as COX defect $(+)$. The estimated doubling time in each sample was also shown. All samples were measured at least in duplicate and averaged. C: Representative images of cellular senescence for cultured myoblasts of both controls and patients. Arrowheads indicate senescence-associated $\beta$-galactosidase (SA- $\beta$-gal)-positive senescent cells. All samples were stained simultaneously with the same period. D: Representative images of apoptotic cell death for cultured myoblasts of both controls and patients. Arrowheads indicate caspase 3-positive apoptotic cells (green). Cell nuclei were costained with Hoechst 33342 (blue). E: Representative images of immunocytochemistry against skeletal muscle tissue-specific markers of myogenin (MYOG) and actin, $\alpha 1$, skeletal muscle (ACTA1) for differentiated myotubes of both controls and patients. On MYOG immunostaining, mitochondria were costained with MitoTracker (green). On ACTA1 immunostaining, cell nuclei were costained with Hoechst 33342 (blue). The error bars indicate means \pm SD of controls (A and $\mathbf{B}) . n=10$ (A and $\mathbf{B}$, controls); $n=5[\mathbf{A}, \operatorname{coX}$ defect $(-)$ ]; $n=2$ $[\mathbf{A}$ and $\mathbf{B}, \mathbf{C O X}$ defect $(+)]$. Scale bars $=50 \mu \mathrm{m}(\mathbf{C}-\mathbf{E})$.

under stable cell growth condition. Nevertheless, no apparent difference in in vitro differentiation propensity of myoblasts into myotubes was confirmed in all patients when compared with controls, which was determined by the results of immunocytochemistry against skeletal muscle tissue-specific markers of myogenin and actin, $\alpha 1$, skeletal muscle (Figure 4E). These results demonstrate that mitochondrial dysfunctions triggered by COX holoenzyme disorganization can induce widespread cellular dysfunctions, but cannot affect skeletal muscle development in patients. The data presenting mitochondrial and cellular biochemical diagnosis in each patient are summarized in Table 1.

\section{Discussion}

In this study, we characterized disease phenotypic differences among patients exhibiting mitochondrial diseases with muscle histopathological COX deficiency by using a comprehensive functional analysis in mitochondria and cells. We demonstrated that widespread mitochondrial and cellular dysfunctions were actually dominated, at least in 
Table 1 Mitochondrial and Cellular Biochemical Diagnosis in Each Patient with Muscle Histopathological COX Deficiency

\begin{tabular}{|c|c|c|c|c|c|c|c|c|c|}
\hline Patient no. & COX function* & $\begin{array}{l}\text { COX } \\
\text { structure }\end{array}$ & ATP level* & ROS level* & $\Delta \Psi \mathrm{m}$ level ${ }^{*}$ & $\begin{array}{l}\text { Cellular } \\
\text { proliferation* }\end{array}$ & $\begin{array}{l}\text { Cellular } \\
\text { senescence }\end{array}$ & $\begin{array}{l}\text { Cellular } \\
\text { apoptosis }\end{array}$ & $\begin{array}{l}\text { Cellular } \\
\text { differentiation }\end{array}$ \\
\hline 10 Controls & $100.0 \pm 27.2$ & Normal & $100.0 \pm 10.3$ & $100.0 \pm 12.2$ & $100.0 \pm 11.2$ & $100.0 \pm 3.9$ & ND & ND & Normal \\
\hline 2 & 46.4 & Normal & 104.8 & 149.6 & 93.9 & 77.3 & ND & ND & Normal \\
\hline 3 & 51.5 & Normal & 130.2 & 122.6 & 117.6 & 89.8 & ND & ND & Normal \\
\hline 4 & 25.6 & Normal & 92.6 & 168.2 & 92.3 & 78.1 & ND & ND & Normal \\
\hline 7 & 66.9 & Normal & 109.7 & 115.2 & 120.8 & 96.0 & ND & ND & Normal \\
\hline
\end{tabular}

COX, cytochrome $c$ oxidase; ND, not detected; ROS, reactive oxygen species; $\Delta \Psi \mathrm{m}$, membrane potential.

*Values are expressed as the percentage against the mean value of 10 controls.

part, by the aberrant COX holoenzyme organization, possibly underlying the variation and the severity in clinical phenotypes of patients. According to these results, we also think it reasonable to classify two obviously different severities in molecular diagnostic criteria of COX deficiency (Figure 5): structurally stable, but functionally mild/moderate defect and severe functional defect with the disrupted COX holoenzyme structure, followed by several mitochondrial and cellular dysfunctions. Patients 5 and 6 are categorized as histopathologically and biochemically severe COX deficiency because of COX holoenzyme disorganization, which must be caused by genetic defects in COXassociating genes. On the other hand, the other patients may be affected by functional abnormality of COX holoenzyme itself or by other unknown physiological abnormalities to apparently induce muscle histopathological COX deficiency as secondary clinical phenotypes. In these cases, it is speculated that disease-causative mutations of muscle

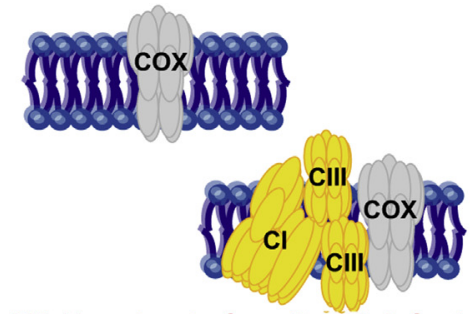

Mild/moderate functional defect

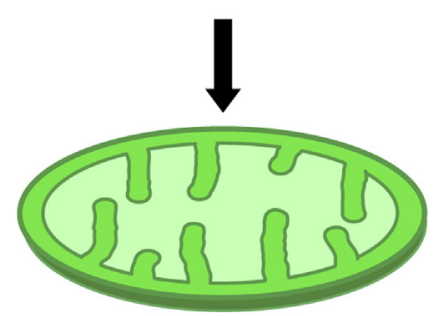

Apparently normal

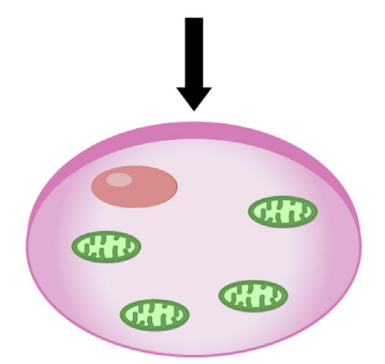

Apparently normal

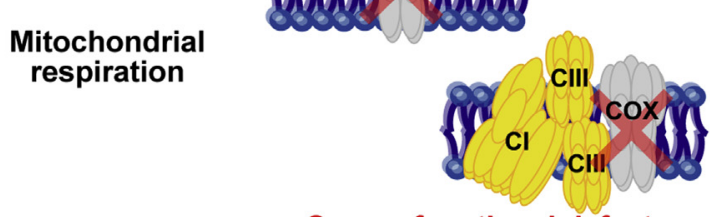

Severe functional defect with COX holoenzyme disorganization

\section{Mitochondrial physiology}

\section{Cellular physiology}

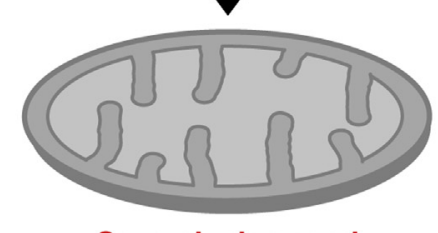

Severely damaged

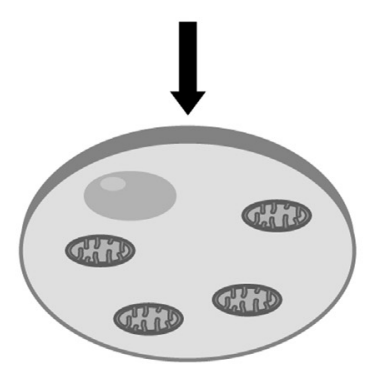

Severely damaged

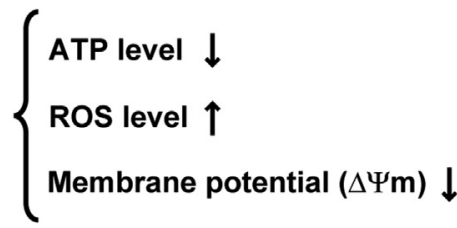

$\left\{\begin{array}{l}\text { Proliferation ability } \downarrow \\ \text { Accelerated scenescence } \\ \text { Induced apoptotic death }\end{array}\right.$

Figure 5 Mitochondrial and cellular phenotypic variation in COX deficiency: A graphical summary. Our in vitro diagnostic approaches successfully demonstrate two obviously different severities in molecular diagnostic criteria of COX deficiency: left column, structurally stable, but functionally mild/moderate defect; and right column, severe functional defect with the disrupted COX holoenzyme structure, followed by several mitochondrial and cellular dysfunctions. 
histopathological COX deficiency patients exhibiting stable COX holoenzyme organization may be in non-COXassociating genes. Therefore, our proposed molecular diagnostic criteria would also be suggestive for effectively exploring the candidate genes, which are responsible for patient-specific pathology, by using next-generation sequencing technology.

The molecular pathomechanism of biochemically severe COX deficiency is summarized as follows: Genetic defects in any COX-associating genes can induce the aberrant COX holoenzyme organization, ${ }^{1}$ and the synthesized but the unassembled COX structural subunits are gradually degraded by some mitochondrial metalloproteases to prevent their accumulation in mitochondrial inner membrane. ${ }^{18}$ That is why lower protein expression levels of several COX structural subunits, despite their stable mRNA syntheses, were observed only in Patients 5 and 6 lacking COX holoenzyme. COX holoenzyme disorganization can induce not only its severe functional defect but also the diminished assembly to form COX-containing respiratory chain supramolecular architectures. In fact, the importance of COX holoenzyme in respiratory chain supercomplexes has been reported, ${ }^{19}$ and the optimized protein ratio of each respiratory chain complex is critical for their supramolecular assembly formation, allowing much higher electron transfer rates in mitochondrial oxidative phosphorylation. ${ }^{20}$ Thus, significant loss of COX holoenzyme induces drastically decreased activity in the production of ATP and thermal energy caused by an insufficient proton electrochemical gradient between mitochondrial matrix and intermembrane space. Mitochondrial respiratory chain complexes are also generally known to increase oxidative stress with their functional defects, and in this case, severely impaired COX holoenzyme integrity seems most likely to affect the increased oxidative stress level. To date, it still remains uncertain whether approximately 2.5 -fold increase of oxidative stress level observed only in Patients 5 and 6 lacking COX holoenzyme is substantially harmful in in vivo mitochondrial physiology. However, the increased oxidative stress level may trigger the accumulated oxidative damages to other mitochondrial enzymes, substrates, lipids, and mtDNA, all of which lead to the depressed overall mitochondrial functions and induce premature senescence at a cell level. In addition, the damaged membrane potential level implies two major mitochondrial abnormalities: transport machinery defects of proteins and substrates essential for mitochondrial biogenesis and bioenergetics and the accelerated leak of freely mobile cytochrome $c$ molecules, as a caspase activator, in mitochondrial electron transport system, followed by the induced apoptotic signaling. Therefore, widespread cellular dysfunctions, including the deteriorated cellular growth, the accelerated cellular senescence, and the induced apoptotic cell death, all of which were observed only in Patients 5 and 6 , are clearly explained by primary COX holoenzyme disorganization and the following secondary mitochondrial dysfunctions.
The relationships between the molecular abnormalities in mitochondrial respiratory chain complexes and their negative contributions to mitochondrial and cellular functions have been proved to be essential for better understandings in mitochondrial medicine. In particular, most parts of mitochondrial diseases are caused by heteroplasmic mutations in mtDNA (wild-type mtDNA and mutant mtDNA coexist within a single cell) and present a wide variety of clinical spectrum among patients, probably because of variations in mutant mtDNA proportions at each tissue and organ level. To date, patients' fibroblasts are mainly used for biochemical analysis. However, such fibroblasts do not always exhibit mitochondrial respiratory defects, most likely because of relatively lower mutant mtDNA proportions than those in the affected tissues and organs of some patients. Although this study does not include mitochondrial disease patients with muscle histopathological COX deficiency, those carrying heteroplasmic mtDNA mutations, our in vitro diagnostic approaches by using patients' myoblasts may be more advantageous, because cells derived from the affected tissues and organs can faithfully recapitulate their cell type-specific pathophysiology in a patient-specific manner. We also believe the use of nonviral, integration-free cellular reprogramming technology ${ }^{21,22}$ to generate disease-relevant induced pluripotent stem cells from patients' fibroblasts or peripheral blood cells can greatly help us to identify bona fide pathomechanism of complex, severe clinical phenotypes in mitochondrial diseases with multiple organ involvements. In fact, several groups and we have recently reported patient-specific induced pluripotent stem cells, those carrying various types of pathogenic mutant mtDNAs as in vitro human mitochondrial disease models, ${ }^{23-29}$ toward possible applications in induced pluripotent stem cell-based drug discovery and regenerative therapeutics. ${ }^{30}$

\section{Conclusions}

Our cell-based in vitro diagnostic approaches documented herein would hold promise for enormous contributions to clinical research for future personalized medicine, which is based on the intrinsic molecular and cellular pathogenic features of each patient exhibiting various types of mitochondrial diseases, including other respiratory chain complex deficiencies and other mitochondrial metabolic enzyme deficiencies.

\section{Acknowledgments}

We thank all patients and their families for participating in this study; Mayuko Kato and Junko Takei (National Center of Neurology and Psychiatry, Tokyo, Japan) for helpful experimental assistance; and Dr. Ichizo Nishino (National Center of Neurology and Psychiatry, Tokyo, Japan) for providing clinical data of muscle histopathology in each patient. 
H.H. conceived the study, designed and performed experiments, analyzed and interpreted data, and wrote the manuscript; and Y.G. analyzed and interpreted data and wrote the manuscript. All authors read and approved the final manuscript.

\section{Supplemental Data}

Supplemental material for this article can be found at http://dx.doi.org/10.1016/j.ajpath.2016.09.003.

\section{References}

1. Shoubridge EA: Cytochrome c oxidase deficiency. Am J Med Genet 2001, 106:46-52

2. Massa V, Fernandez-Vizarra E, Alshahwan S, Bakhsh E, Goffrini P, Ferrero I, Mereghetti P, D’Adamo P, Gasparini P, Zeviani M: Severe infantile encephalomyopathy caused by a mutation in COX6B1, a nucleus-encoded subunit of cytochrome c oxidase. Am J Hum Genet 2008, 82:1281-1289

3. Indrieri A, van Rahden VA, Tiranti V, Morleo M, Iaconis D, Tammaro R, D'Amato I, Conte I, Maystadt I, Demuth S, Zvulunov A, Kutsche K, Zeviani M, Franco B: Mutations in COX7B cause microphthalmia with linear skin lesions, an unconventional mitochondrial disease. Am J Hum Genet 2012, 91:942-949

4. Keightley JA, Hoffbuhr KC, Burton MD, Salas VM, Johnston WS, Penn AM, Buist NR, Kennaway NG: A microdeletion in cytochrome c oxidase (COX) subunit III associated with COX deficiency and recurrent myoglobinuria. Nat Genet 1996, 12:410-416

5. Comi GP, Bordoni A, Salani S, Franceschina L, Sciacco M, Prelle A, Fortunato F, Zeviani M, Napoli L, Bresolin N, Moggio M, Ausenda CD, Taanman JW, Scarlato G: Cytochrome c oxidase subunit I microdeletion in a patient with motor neuron disease. Ann Neurol 1998, 43:110-116

6. Hanna MG, Nelson IP, Rahman S, Lane RJ, Land J, Heales S, Cooper MJ, Schapira AH, Morgan-Hughes JA, Wood NW: Cytochrome c oxidase deficiency associated with the first stop-codon point mutation in human mtDNA. Am J Hum Genet 1998, 63: 29-36

7. Bruno C, Martinuzzi A, Tang Y, Andreu AL, Pallotti F, Bonilla E, Shanske S, Fu J, Sue CM, Angelini C, DiMauro S, Manfredi G: A stop-codon mutation in the human mtDNA cytochrome c oxidase I gene disrupts the functional structure of complex IV. Am J Hum Genet 1999, 65:611-620

8. Clark KM, Taylor RW, Johnson MA, Chinnery PF, ChrzanowskaLightowlers ZM, Andrews RM, Nelson IP, Wood NW, Lamont PJ, Hanna MG, Lightowlers RN, Turnbull DM: An mtDNA mutation in the initiation codon of the cytochrome $\mathrm{c}$ oxidase subunit II gene results in lower levels of the protein and a mitochondrial encephalomyopathy. Am J Hum Genet 1999, 64:1330-1339

9. Rahman S, Taanman JW, Cooper JM, Nelson I, Hargreaves I, Meunier B, Hanna MG, García JJ, Capaldi RA, Lake BD, Leonard JV, Schapira AH: A missense mutation of cytochrome oxidase subunit II causes defective assembly and myopathy. Am J Hum Genet 1999, 65: $1030-1039$

10. Tiranti V, Corona P, Greco M, Taanman JW, Carrara F, Lamantea E, Nijtmans L, Uziel G, Zeviani M: A novel frameshift mutation of the mtDNA COIII gene leads to impaired assembly of cytochrome c oxidase in a patient affected by Leigh-like syndrome. Hum Mol Genet 2000, 9:2733-2742

11. Horvath R, Kemp JP, Tuppen HA, Hudson G, Oldfors A, Marie SK, Moslemi AR, Servidei S, Holme E, Shanske S, Kollberg G, Jayakar P, Pyle A, Marks HM, Holinski-Feder E, Scavina M, Walter MC, Coku J, Günther-Scholz A, Smith PM, McFarland R, Chrzanowska-
Lightowlers ZM, Lightowlers RN, Hirano M, Lochmüller H, Taylor RW, Chinnery PF, Tulinius M, DiMauro S: Molecular basis of infantile reversible cytochrome c oxidase deficiency myopathy. Brain 2009, 132:3165-3174

12. Mimaki M, Hatakeyama H, Komaki H, Yokoyama M, Arai $\mathrm{H}$, Kirino Y, Suzuki T, Nishino I, Nonaka I, Goto Y: Reversible infantile respiratory chain deficiency: a clinical and molecular study. Ann Neurol 2010, 68:845-854

13. Akanuma J, Muraki K, Komaki H, Nonaka I, Goto Y: Two pathogenic point mutations exist in the authentic mitochondrial genome, not in the nuclear pseudogene. J Hum Genet 2000, 45:337-341

14. Tanaka M, Takeyasu T, Fuku N, Li-Jun G, Kurata M: Mitochondrial genome single nucleotide polymorphisms and their phenotypes in the Japanese. Ann N Y Acad Sci 2004, 1011:7-20

15. Trounce IA, Kim YL, Jun AS, Wallace DC: Assessment of mitochondrial oxidative phosphorylation in patient muscle biopsies, lymphoblasts, and transmitochondrial cell lines. Methods Enzymol 1996, 264:484-509

16. Schägger H: Tricine-SDS-PAGE. Nat Protoc 2006, 1:16-22

17. Wittig I, Braun HP, Schägger H: Blue native PAGE. Nat Protoc 2006, $1: 418-428$

18. Arnold I, Langer T: Membrane protein degradation by AAA proteases in mitochondria. Biochim Biophys Acta 2002, 1592:89-96

19. Schafer E, Seelert H, Reifschneider NH, Krause F, Dencher NA, Vonck J: Architecture of active mammalian respiratory chain supercomplexes. J Biol Chem 2006, 281:15370-15375

20. Schägger H, Pfeiffer K: Supercomplexes in the respiratory chains of yeast and mammalian mitochondria. EMBO J 2000, 19: $1777-1783$

21. Okita K, Matsumura Y, Sato Y, Okada A, Morizane A, Okamoto S, Hong H, Nakagawa M, Tanabe K, Tezuka K, Shibata T, Kunisada T, Takahashi M, Takahashi J, Saji H, Yamanaka S: A more efficient method to generate integration-free human iPS cells. Nat Methods 2011, 8:409-412

22. Okita K, Yamakawa T, Matsumura Y, Sato Y, Amano N, Watanabe A, Goshima N, Yamanaka S: An efficient nonviral method to generate integration-free human-induced pluripotent stem cells from cord blood and peripheral blood cells. Stem Cells 2013, $31: 458-466$

23. Fujikura J, Nakao K, Sone M, Noguchi M, Mori E, Naito M, Taura D, Harada-Shiba M, Kishimoto I, Watanabe A, Asaka I, Hosoda K, Nakao K: Induced pluripotent stem cells generated from diabetic patients with mitochondrial DNA A3243G mutation. Diabetologia 2012, 55:1689-1698

24. Cherry AB, Gagne KE, McLoughlin EM, Baccei A, Gorman B, Hartung O, Miller JD, Zhang J, Zon RL, Ince TA, Neufeld EJ, Lerou PH, Fleming MD, Daley GQ, Agarwal S: Induced pluripotent stem cells with a mitochondrial DNA deletion. Stem Cells 2013, 31 $1287-1297$

25. Folmes CD, Martinez-Fernandez A, Perales-Clemente E, Li X, McDonald A, Oglesbee D, Hrstka SC, Perez-Terzic C, Terzic A, Nelson TJ: Disease-causing mitochondrial heteroplasmy segregated within induced pluripotent stem cell clones derived from a patient with MELAS. Stem Cells 2013, 31:1298-1308

26. Hämäläinen RH, Manninen T, Koivumäki H, Kislin M, Otonkoski T, Suomalainen A: Tissue- and cell-type-specific manifestations of heteroplasmic mtDNA 3243A $>$ G mutation in human induced pluripotent stem cell-derived disease model. Proc Natl Acad Sci U S A 2013, 110:E3622-E3630

27. Kodaira M, Hatakeyama H, Yuasa S, Seki T, Egashira T, Tohyama S, Kuroda Y, Tanaka A, Okata S, Hashimoto H, Kusumoto D, Kunitomi A, Takei M, Kashimura S, Suzuki T, Yozu G, Shimojima M, Motoda C, Hayashiji N, Saito Y, Goto Y, Fukuda K: Impaired respiratory function in MELAS-induced pluripotent stem cells with high heteroplasmy levels. FEBS Open Bio 2015, 5:219-225

28. Hatakeyama H, Katayama A, Komaki H, Nishino I, Goto Y: Molecular pathomechanisms and cell-type-specific disease phenotypes of 
MELAS caused by mutant mitochondrial tRNA(Trp). Acta Neuropathol Commun 2015, 3:52

29. Ma H, Folmes CD, Wu J, Morey R, Mora-Castilla S, Ocampo A, Ma L, Poulton J, Wang X, Ahmed R, Kang E, Lee Y, Hayama T, Li Y, Van Dyken C, Gutierrez NM, Tippner-Hedges R, Koski A, Mitalipov N, Amato P, Wolf DP, Huang T, Terzic A, Laurent LC,
Izpisua Belmonte JC, Mitalipov S: Metabolic rescue in pluripotent cells from patients with mtDNA disease. Nature 2015, 524:234-238

30. Hatakeyama H, Goto Y: Heteroplasmic mitochondrial DNA mutations and mitochondrial diseases: toward iPSC-based disease modeling, drug discovery, and regenerative therapeutics. Stem Cells 2016, 34: 801-808 